\title{
In situ observation of single-molecule surface reactions from low to high affinities
}

\author{
Eugene Kim, Martin D. Baaske, and Frank Vollmer* \\ Max Planck Institute for the Science of Light, Laboratory of Nanophotonics and \\ Biosensing, Günther-Scharowsky-Straße 1, 91058 Erlangen \\ E-mail: frank.vollmer@mpl.mpg.de
}

\begin{abstract}
Understanding reactions occurring between ligand molecules and nanomaterial surfaces is essential in the field of nanoscience. The conventional methods for characterizing such surface-based reactions allow only for the analysis of the end product of a reaction, although the reaction path proceeds through the transient interaction of reactants and with kinetics dependent on environmental parameters. Here we study single molecule reaction kinetics associated with gold nanoparticle surfaces in an aqueous medium by utilizing whispering-gallery-mode microcavity sensors. Our approach resolves transient as well as permanent interaction kinetics of ligand molecules at the nanoparticle interface in situ, over a broad range of affinities and even under conditions where no net product is formed. This enables us to monitor and characterize reactions during the entire procedure of a bottom-up surface modification, ranging from the deposition of ligands to the confirmation of their functionality. We demonstrate this prospect by studying surface reaction kinetics with respect to the species of ligand head groups, tethered molecules, and inhibitors in addition to subsequent bio-specific reactions between tethered molecules and analytes.
\end{abstract}

${ }^{*}$ To whom correspondence should be addressed 


\section{Introduction}

Understanding molecular interactions at nanomaterial surfaces and engineering their surface properties towards a desired profile is of crucial importance in the field of nanoscience. The chemical reactions occurring between nanomaterial surfaces and ligand molecules constitute the basis for stabilization, ${ }^{1}$ directional growth, ${ }^{2}$ and biocompatible functionalization ${ }^{3}$ of nanomaterials and, therefore, have been thoroughly investigated over the past decades. To gain a general understanding of ligand surface reactions, however, is not trivial since they not only depend on the species of the ligand head groups and surface atoms, but also on the molecules that are tethered to the head groups as well as the environmental conditions. A variety of characterization tools, including nanoscale imaging, spectroscopy, and manipulation techniques, are available for studying specific aspects of such surface reactions. ${ }^{4-6}$ Most of these methods, on the other hand, only allow for the analysis of resultants and hence do not provide feedback on the reaction kinetics if, on average, there is no product formed. This leaves a gap in the low affinity regime of a reaction (where no net product is formed), thus requiring some guesswork in order to find conditions that may raise the yield to or above a discernible level. Here we show that our recently established optical microcavity based sensor can bridge this gap. This sensor system is capable of monitoring both transient and permanent ligand surface interactions in situ and with single-molecule resolution. It hence allows us to study reaction kinetics on nanomaterial surfaces over a broad range of affinities, which, in turn, covers all aspects of a surface modification process from the deposition of ligand molecules to the confirmation of their functionality.

In this work we specifically study the reaction kinetics of two different ligand head groups (i.e. thiol and amine) with nanorod (NR) surface gold atoms in an aqueous solution. We study how the surface reactions evolve from low to high affinity with varying environmental conditions, such as the $\mathrm{pH}$ and electrolyte concentration. In-depth statistical analysis of these single-molecule surface reactions allows us to determine the associated rate constants and to gain further insights into the characteristics of each specific reaction. We also inves- 
tigate the influence of different molecules tethered to the head groups during the ligand-NR surface reactions. The biofunctional groups of the tethered molecules allow us to confirm the functionality of the ligand-modified NRs by monitoring their specific biological reaction. Lastly, we study the surface reaction kinetics in the presence of inhibitors - another regime which is inaccessible to ensemble averaged characterization tools due to its extremely low yield of reaction products.

\section{Results and discussion}

\section{Method for detecting surface reaction kinetics}
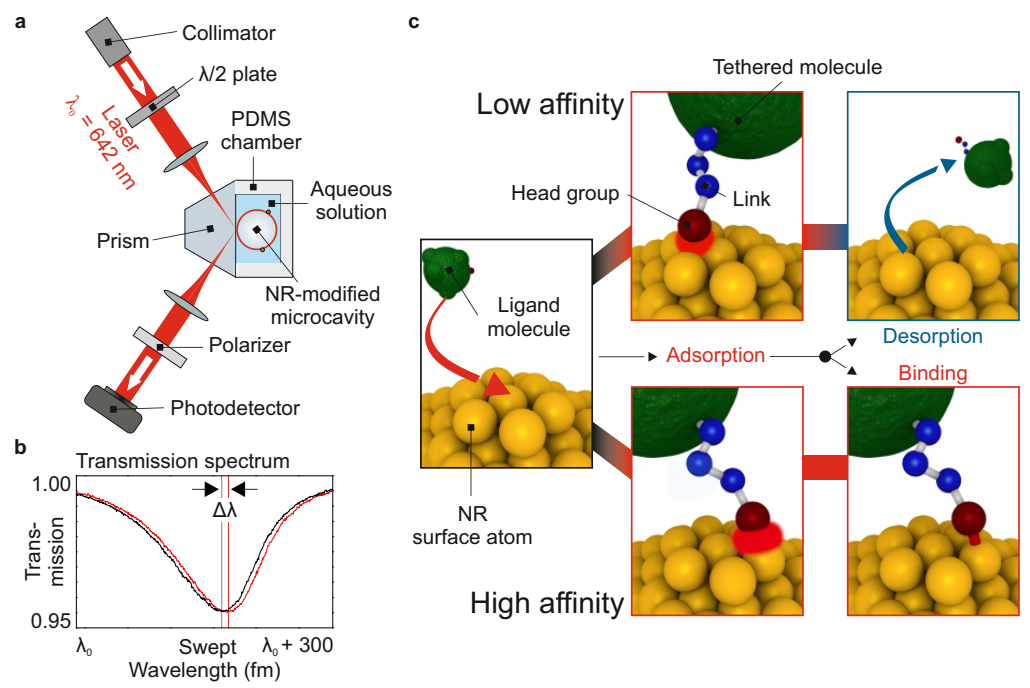

d
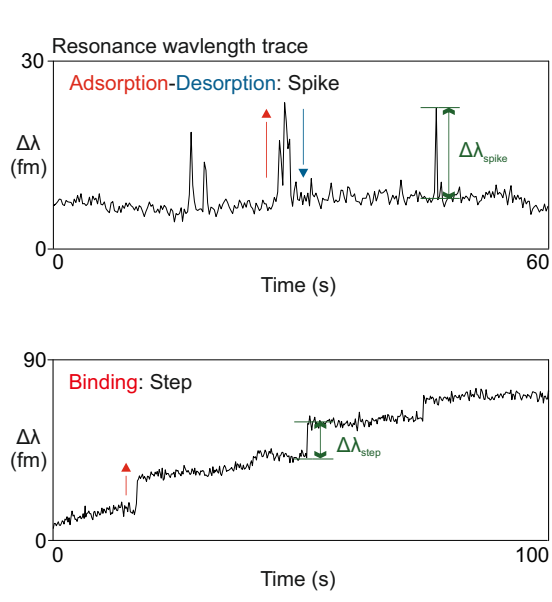

Figure 1: Experimental method and reaction types. (a) Schematic of the prism based microcavity sensor setup. (b) Example of a transmission spectrum for a WGM resonance before (black) and after (red) the red shift caused by the binding of a ligand molecule. (c) Sketch of the two types of ligand-NR surface reactions and (d) the corresponding resonance traces exhibiting spike and step signals. In the low affinity regime, the head group of the ligand molecule reacts transiently with the gold surface (adsorption-desorption), causing a spike pattern. In the high affinity regime, the head group covalently binds to a gold atom, causing a step pattern.

Our method achieves single-molecule sensitivity (Supplementary Section 3) via the cou- 
pling between the optical whispering gallery modes (WGMs) of a microcavity and the surface plasmon resonances (SPRs) of gold NRs immobilized on the cavity's surface. The amplitude of the WGM sensor signal increases with increasing proximity of the analyte to the NRs, making our system an ideal platform for studying chemical reactions occurring at the NR surface. The experimental setup used for the single-molecule study of surface reaction kinetics is depicted in Fig. 1a. A wavelength-tunable CW laser $\left(\lambda_{0} \approx 642 \mathrm{~nm}\right)$ is coupled into a NR-modified fused silica microcavity via frustrated total internal reflection at the prism surface as to excite WGMs. A half-wave plate and a polarizer are used to selectively excite and detect TE and TM modes of the cavity. WGMs are detected in the form of Lorentzian dips in the transmission spectra obtained by sweeping the laser wavelength at a rate of $50 \mathrm{~Hz}$. The NRs immobilized at the surface of the cavity serve two purposes: 1) Local enhancement of the electric field intensity at the sensing site as to enable single-molecule sensitivity ${ }^{7-9}$ and 2) provision of gold atom reactants in the surface reactions. A ligand molecule that interacts with the surface gold atoms within these sensing sites perturbs the WGMs and therefore induces a red shift in their resonance wavelength (Fig. 1b). The observed ligand surface reactions are classified into two different types. The first is transient, indicating a non-zero yet low ligand surface affinity. Possible processes responsible for the transient reaction are either physical adsorption and the consequent desorption of ligands at the surface or the formation of unstable, short-lived bonds between the ligand head groups and the surface atoms (upper illustration of Fig. 1c). These transient reactions are recognized by our sensor system as spikes in the resonance position traces (top subfigure of Fig. 1d). The second type of reaction involves binding, correlated to a high affinity of the ligand surface reaction. This binding is due to the formation of strong chemical bonds between the surface atoms and the ligand head groups (lower illustration of Fig. 1c). Binding type reactions are recognized as discrete steps in the resonance position traces (bottom subfigure of Fig. 1d). The ability of our system to observe transient reactions enables us to study reaction kinetics in a regime where ensemble based techniques are otherwise unable to provide any quantitative 
information due to insufficient reaction product formation. Furthermore, one can obtain direct feedback regarding the influence of environmental conditions on the reaction kinetics to improve the yield, as the method is in situ and reactions are observed in real time. This will be demonstrated in the following sections for different classes of reactions occurring at or near to the surface of the NRs.

\section{In situ observation of reaction kinetics}

The reactions covered in this work are shown along with the corresponding sensor signals in Fig. 2. Chemical reactions between the thiol as well as amine ligand head groups and NR surface gold atoms have been investigated from the low (Fig. 2a) to high (Fig. 2b) affinity regime. The different affinities of the respective reactions have been controlled by adjusting environmental conditions, such as $\mathrm{pH}$ and electrolyte concentration. In addition, the influence of the species of tethered molecules on the monitored surface reactions has been investigated by comparing two different molecules: single stranded DNA oligonucleotides (26 bases long) and biotinylated polyethylenglycol (PEG) (Fig. 2c). Two well-known biological reactions (i.e. antigen-antibody reaction, DNA hybridization) have also been studied and compared against the corresponding ligand surface reactions in order to confirm the surface functionality of the ligand-modified NRs (Fig. 2d). The ability of our sensor to detect transient reactions also enables us to monitor how efficiently a reaction can be hindered or halted. We address these aspects by studying the kinetics of the reaction between thiol groups and NR surface atoms in the presence of inhibitor molecules, sodium dodecyl sulfate (SDS), in Fig. $2 \mathrm{e}$.

\section{Ligand surface reactions from low to high affinities}

Ligand-NR surface reactions in the low affinity regime exclusively exhibit transient reactions for both thiol and amine groups (Fig. 2a). To induce transient reactions for both head groups, we adjust the $\mathrm{pH}$ of the aqueous solution. In the case of the thiol groups, reducing 

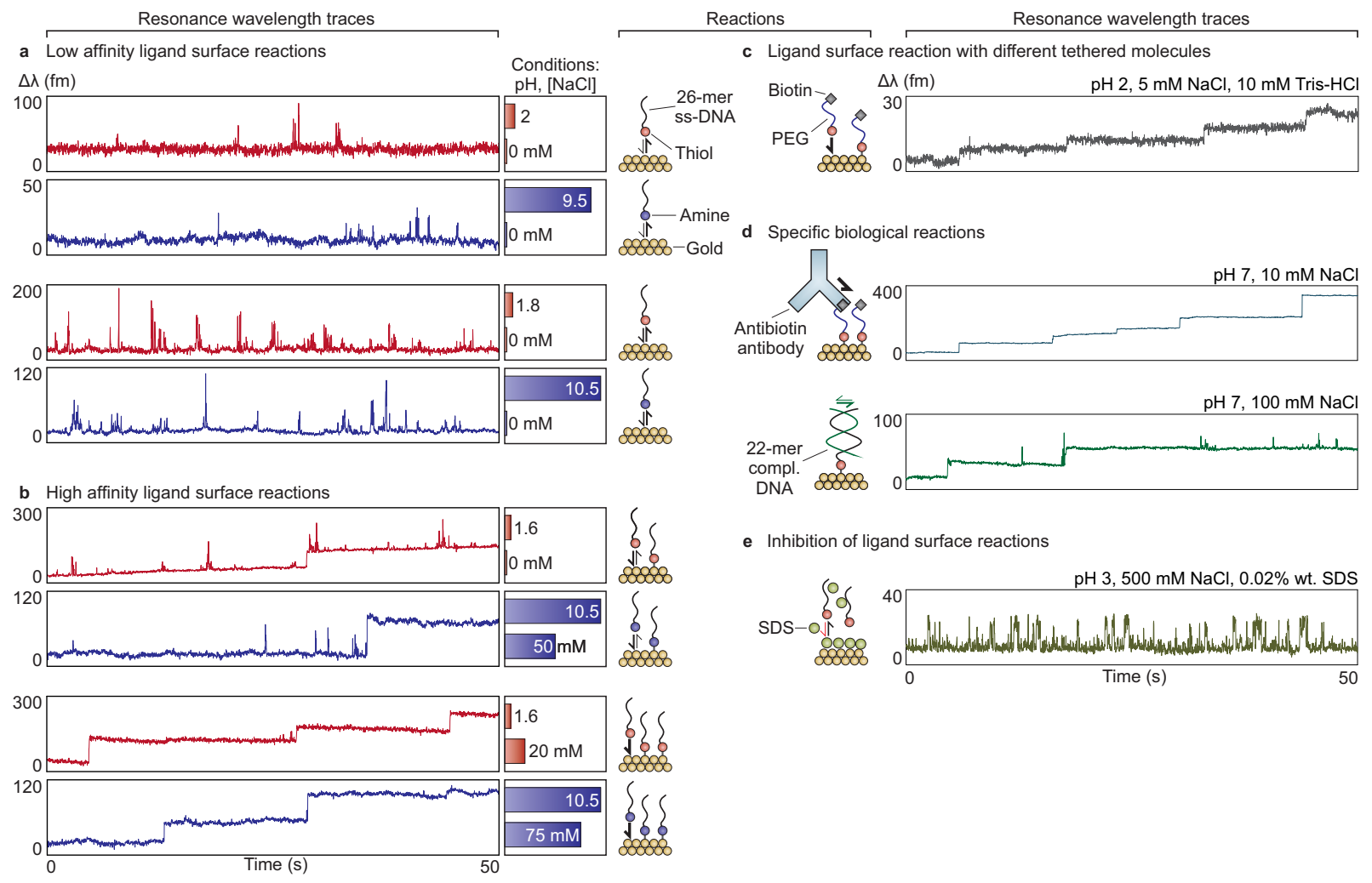

e Inhibition of ligand surface reactions

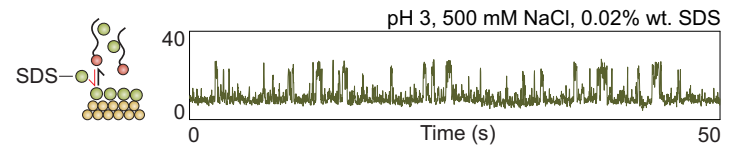

Figure 2: Overview of monitored reactions. Each box contains a representative trace obtained by monitoring the corresponding surface reactions, which are depicted in between. Sensor signals for reactions between thiol as well as amine ligand head groups and NR surfaces evolving from low to high affinity are displayed in $(a, b)$. At low affinities, spike signals appear (a, top) and occur more frequently (a, bottom) as the environmental conditions become more favorable for the reaction. In the high affinity regime, steps occur alongside spikes (b, top), whereas under more optimal conditions the steps dominate the spectrum (b, bottom). (c) Thiol-NR surface reactions for a different tethered molecule (biotinylated PEG). (d) The subsequent bio-specific reactions observed between tethered molecules and analytes: anti-biotin antibody binding to biotin (top) and single-stranded DNA hybridizing with its complementary strand (bottom). (e) Signals from thiol-NR surface reactions monitored in the presence of SDS. 
Reaction kinetics at low affinity
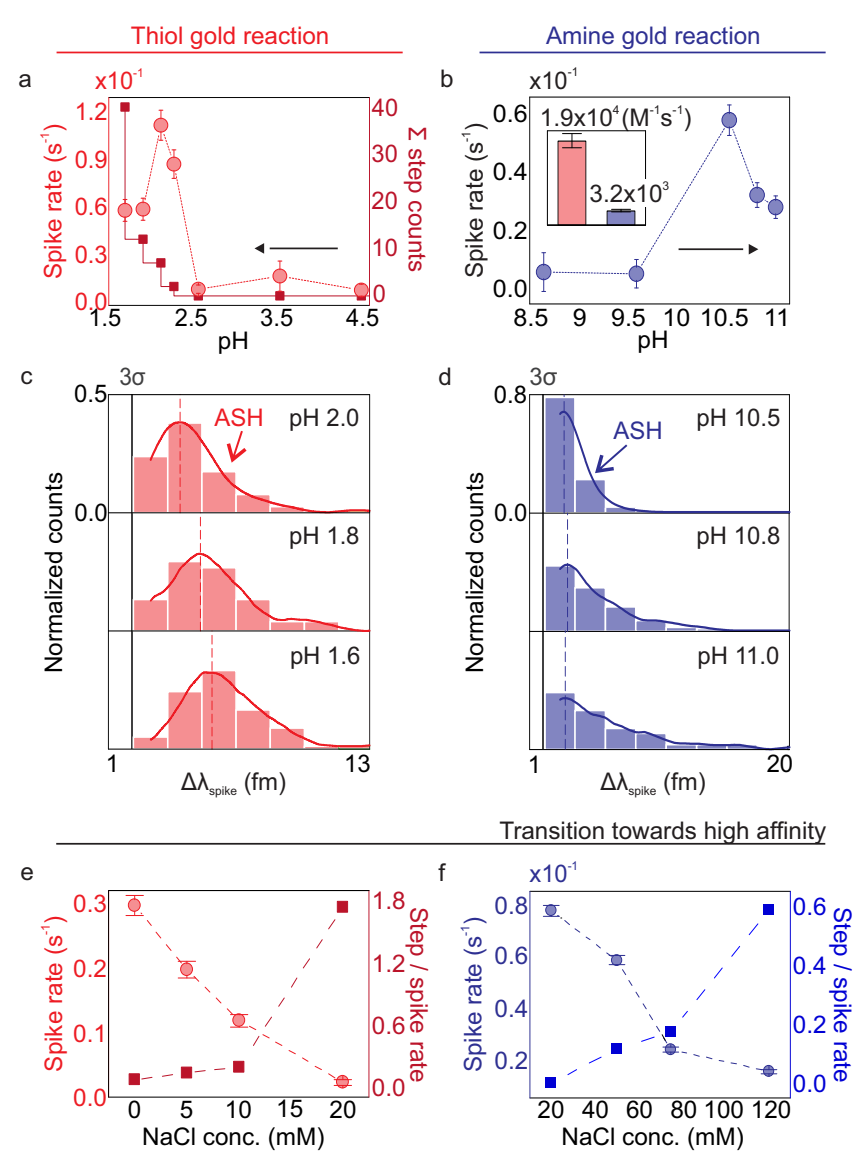

Figure 3: Reaction kinetics of thiol and amine head groups with NR surfaces from low to high affinities. Spike rates and cumulative step counts for (a) thiol and (b) amine head groups, obtained as the $\mathrm{pH}$ is stepwise changed in the direction indicated by the black arrows. The inset of (b) shows the direct comparison between spike rates for thiol (red) and amine (blue) groups, normalized by the ligand concentration and number of NRs. Distributions of spike heights $\Delta \lambda_{\text {spike }}$ obtained at different $\mathrm{pH}$ values for (c) thiol and (d) amine groups displayed as histograms (columns) and their corresponding averaged shifted histograms (ASH). Dashed straight lines indicate the peak positions and grey solid lines indicate the spike detection limit, 3 times the noise level $\sigma$. Evolution of the spike rates and the ratio of step to spike rate when increasing $\mathrm{NaCl}$ concentration for (e) thiol and (f) amine. 
the $\mathrm{pH}$ from 7 to 3 does not yield a significant increase in the spike rate. Further reduction below 2.5, however, induces spikes with comparably high frequencies. This trend continues with the reduction of the $\mathrm{pH}$ to 2 . As we further decrease the $\mathrm{pH}$ to 1.8 we begin to observe step events (Fig. 3a), occurring simultaneously with a drop in the spike rate as the total number of surface atoms available for the transient reaction is depleted due to irreversible thiol-gold bond formation. In the case of the amine groups, an increase of the $\mathrm{pH}$ beyond 7 is required in order to observe the initial transient reactions. At a $\mathrm{pH} 8.5$, spike events appear at a relatively low rate. This greatly increases as the $\mathrm{pH}$ rises towards 10.5. Even larger $\mathrm{pH}$ values cause the spike rate to drop again, with no simultaneous step event occurrence (Fig. 3b). The maximal spike rates, normalized by the ligand concentration and number of NRs, obtained for thiol and amine (Inset of Fig. 3b) differ by a factor of $\sim 6$ despite the molecules' similar molecular masses (8.29 and $8.28 \mathrm{kDa}$, respectively). This disparity most likely originates from the large difference in binding energies for gold and sulfur $(\sim 50$ $\mathrm{kcal} / \mathrm{mol})$ as well as gold and nitrogen $(\sim 3-9 \mathrm{kcal} / \mathrm{mol}),{ }^{10,11}$ which in turn determines the number of overall available reaction sites per NR and the consequent rates. The influence of the environmental $\mathrm{pH}$ on the transient reactions also manifests itself in the distributions of the average spike heights $\Delta \lambda_{\text {spike }}$ (Fig. 3c and 3d). For both head groups we observe a clear shift in the spike height distributions towards higher values as we tune the $\mathrm{pH}$ towards lower (for thiol) and higher (for amine) values. This indicates that lower (higher) $\mathrm{pH}$ values allow the thiol (amine) groups to come closer to the surface of the NRs, thus causing spike signals with larger amplitudes. In terms of the thiol groups this might be due to reduced repulsion between the ligand molecule and the $\mathrm{NR}$ at low $\mathrm{pH}$, favoring the adsorption of thiol groups. As for the amine groups, the reaction is instead hindered from low to neutral $\mathrm{pH}$ as they remain in their nonreactive, charged form $\left(\mathrm{NH}_{3}^{+}\right)$. At a high $\mathrm{pH}$, most of these amine groups are deprotonated $\left(\mathrm{NH}_{2}\right)$ and the lone electron pair of the nitrogen atom can consequently interact with gold atoms. ${ }^{12}$ Starting from the optimal $\mathrm{pH}$ values, equal to 2 for thiol and 10.5 for amine, the affinity between both head groups and the surface gold atoms 
can be further increased by the introduction of sodium chloride ( $\mathrm{NaCl})$ into the solution. An increase of the $\mathrm{NaCl}$ concentration induces a gradual transition from predominantly transient to near-exclusive binding type reactions (Fig. 3e and 3f). This results in a sudden increase in the ratio of the step to spike rate at around $10 \mathrm{mM} \mathrm{NaCl}$ for thiol (Fig. 3e) and $75 \mathrm{mM}$ for amine (Fig. 3f), while the overall spike rate drops as a consequence of binding-induced depletion of reaction sites.

\section{Ligand surface reactions at high affinity}
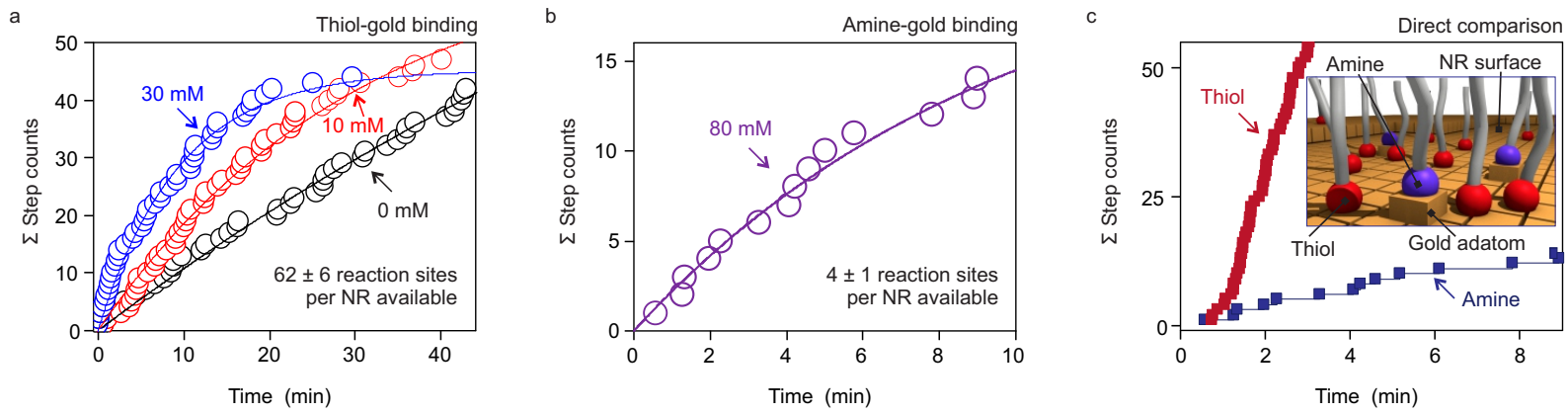

Figure 4: Ligand surface binding kinetics at high affinity. Time dependent cumulative step counts (circles) obtained for (a) thiol-gold binding and (b) amine-gold binding. Numbers to which arrows are attached indicate the $\mathrm{NaCl}$ concentrations that were used and solid lines are the fitted saturation curves. (c) Direct comparison between the cumulative step counts obtained by amine-gold and the subsequent thiol-gold binding on the same NRs. The inset of (c) shows a sketch of the thiol and amine groups occupying different binding sites.

In order to elaborate on the reaction kinetics in high affinity regime, we will now focus on the binding type events. With only a limited number of surface atoms available within the sensing sites of the NRs, the rate at which binding events are observed drops over time as more and more surface atoms are occupied by ligand molecules. This allows for the extraction of both $k_{b i n d}$, the binding rate per reaction site, and $N_{0}$, the number of available reaction sites within the sensing sites at the start of the measurement, by fitting the saturation function $N(t)=N_{0}\left(1-e^{-k_{b i n d} t}\right)$ to the measured time dependent cumulative step counts $N(t)$ (Fig. 
$4 \mathrm{a}$ and $4 \mathrm{~b}$ ). For the thiol-gold reaction (Fig. 4a) at $\mathrm{pH} 1.6$ and $\mathrm{NaCl}$ concentrations of 0 , 10 , and $30 \mathrm{mM}$ we find $k_{\text {bind }}$ values of $0.14,0.54$, and $1.8\left(\times 10^{3} \mathrm{~s}^{-1} \mathrm{M}^{-1}\right)$, which are in good agreement with the thiol adsorption rates measured in ensemble averaging techniques. ${ }^{13,14}$ For the amine group we obtain a $k_{b i n d}$ of $1.5\left(\times 10^{3} \mathrm{~s}^{-1} \mathrm{M}^{-1}\right)$ at a $\mathrm{pH}$ of 10.5 and a $\mathrm{NaCl}$ concentration of $80 \mathrm{mM}$ (Fig. 4b). Both head groups, despite the significant difference in the binding energies of gold-sulfur and gold-nitrogen, exhibit similar binding rates with gold - a finding that is in agreement with previous bulk measurements. ${ }^{15}$ The extracted numbers of available reaction sites (within the sensing sites), however, exhibit a significant difference as we observe an average of $62 \pm 6$ sites per NR for thiol but only $4 \pm 1$ for amine. The overall lower event rates for amine-modified oligonucleotides, as compared to their thiol-modified counterparts, thus originate from the overall lower number of reaction sites. Also, our data suggests that in comparison to the thiol-gold reaction, less and possibly different surface atoms are involved in the amine-gold reaction, as we observe significant counts of thiol-gold binding events even if the same NRs were fully saturated with aminemodified oligonucleotides in a previous step (Fig. 4c). Additionally, steps towards shorter wavelengths did not appear, as is expected for thiol groups cleaving the amine-gold bond if both would compete for the same binding sites. Our findings agree with previous studies which suggest that the amine groups selectively bind to low coordinated surface gold atoms (adatoms or surface roughness), whereas the thiol groups preferentially bind to flat (100) and (111) gold surfaces ${ }^{16-18}$ (inset of Fig. 4c).

\section{Effects of tethered molecules}

So far, we have only focused on the reaction between ligand head groups and gold surfaces while neglecting the influence of tethered molecule properties, such as the overall mass and the magnitude as well as polarity of their surface charge, on the reaction kinetics. We hence proceed to compare the same type of surface reaction (i.e. thiol-gold) for two different tethered species: thiol-conjugated polyethylene glycol (PEG) biotin molecules and thiol- 

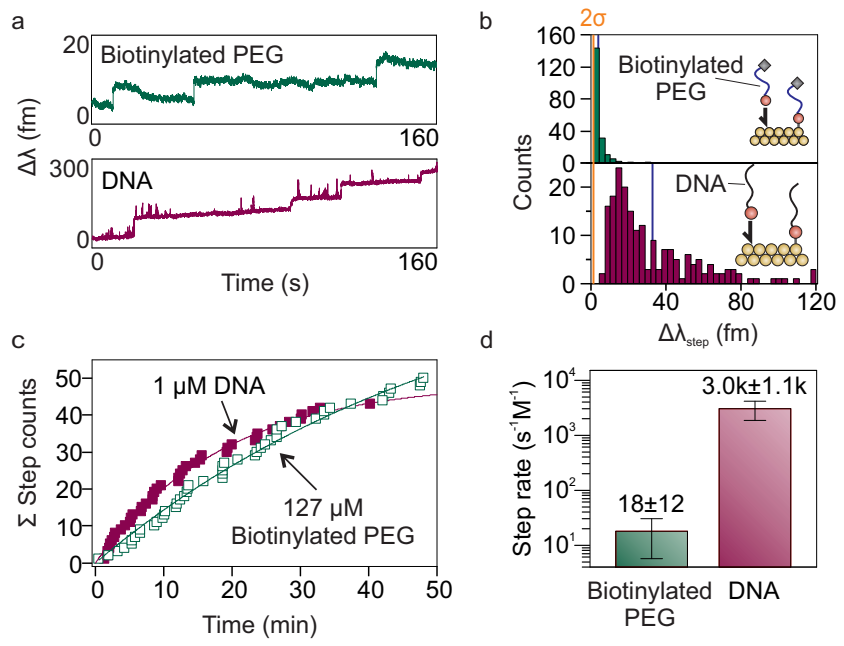

Figure 5: Influence of tethered molecules on thiol-NR surface reactions. (a) Sample resonance traces with signals due to biotinylated PEG (top) and ss-DNA oligonucleotides (bottom) tethered to thiol groups. (b) Step size distributions (histogram) and average step sizes (solid line) reflecting the significant difference in the molecular mass of both ligands. (c) Comparison between the saturation curves fitted to the cumulative step counts obtained for both ligand molecules in solutions with identical $\mathrm{NaCl}$ concentrations (i.e. $10 \mathrm{mM}$ ) and microspheres of similar diameters. (d) Comparison between the average step rates obtained by averaging over the results of 6 measurements for each ligand. The rates are normalized by the number of used NRs and the ligand concentration. 
modified DNA oligonucleotides (Fig. 5). To achieve a high yield of binding for PEG biotin, we have adapted a protocol from ${ }^{19}(\mathrm{pH} 2, \mathrm{NaCl} 5-10 \mathrm{mM}$, tris- $\mathrm{HCl} 10 \mathrm{mM})$ as we did not observe a substantial number of events without the introduction of tris- $\mathrm{HCl}$. In contrast to the DNA oligonucleotides, spike events were seldom seen and the signal was principally comprised of step events (Fig. 5a). This possibly indicates that the transient interactions may be too short to resolve with the $20 \mathrm{~ms}$ time resolution of our system. The step size distributions obtained for both tethered molecules and their average values directly reflect the difference in their respective molecular mass (Fig. 5b). For the PEG biotin (with molecular mass $=0.78 \mathrm{kDa})$ we obtained one-sided decay $\left(\overline{\Delta \lambda}_{\text {step }}=4.2 \mathrm{fm}\right)$, indicative of a good fraction of events possibly failing to exceed the noise level $\sigma$ by at least a factor of 2 , whereas the distribution obtained for the DNA oligonucleotides (with molecular mass $=8.28$ $\mathrm{kDa}$ ) exhibits a clear peak in excess of this $2 \sigma$ limit and an overall higher step amplitude $\left(\overline{\Delta \lambda}_{\text {step }}=32.6 \mathrm{fm}\right)$. By fitting saturation curves to the time dependent cumulative step counts for $10 \mathrm{mM} \mathrm{NaCl}$ common to both tethered molecules (Fig. 5c), we determine binding rates of $2.4 \mathrm{~s}^{-1} \mathrm{M}^{-1}$ and $4.7 \times 10^{2} \mathrm{~s}^{-1} \mathrm{M}^{-1}$ per site and the number of observable binding sites of $87 \pm 6$ and $72 \pm 1$ per NR for PEG biotin and DNA-oligonucleotides, respectively. This shows that the binding affinity of the thiol groups to the gold surface strongly depends on the type of molecule they are tethered to. This is also reflected by the two order of magnitude difference in the average step event rates per NR, as obtained from the results of 6 experiments for each case (Fig. 5d).

\section{Sequential reactions and the influence of inhibitors}

In biomedical research, nanoparticles (NPs) are generally tethered with a diverse set of ligand molecules (proteins, DNA, polymers, dyes, etc.) for the study of specific interactions with analyte molecules. Unfortunately, ligand-NP surface reactions are only generally considered as a tool to functionalize NPs and the quantitative study of the interaction between ligands and analytes (2nd stage) with respect to ligand-NP surface reactions (1st stage) have rarely 

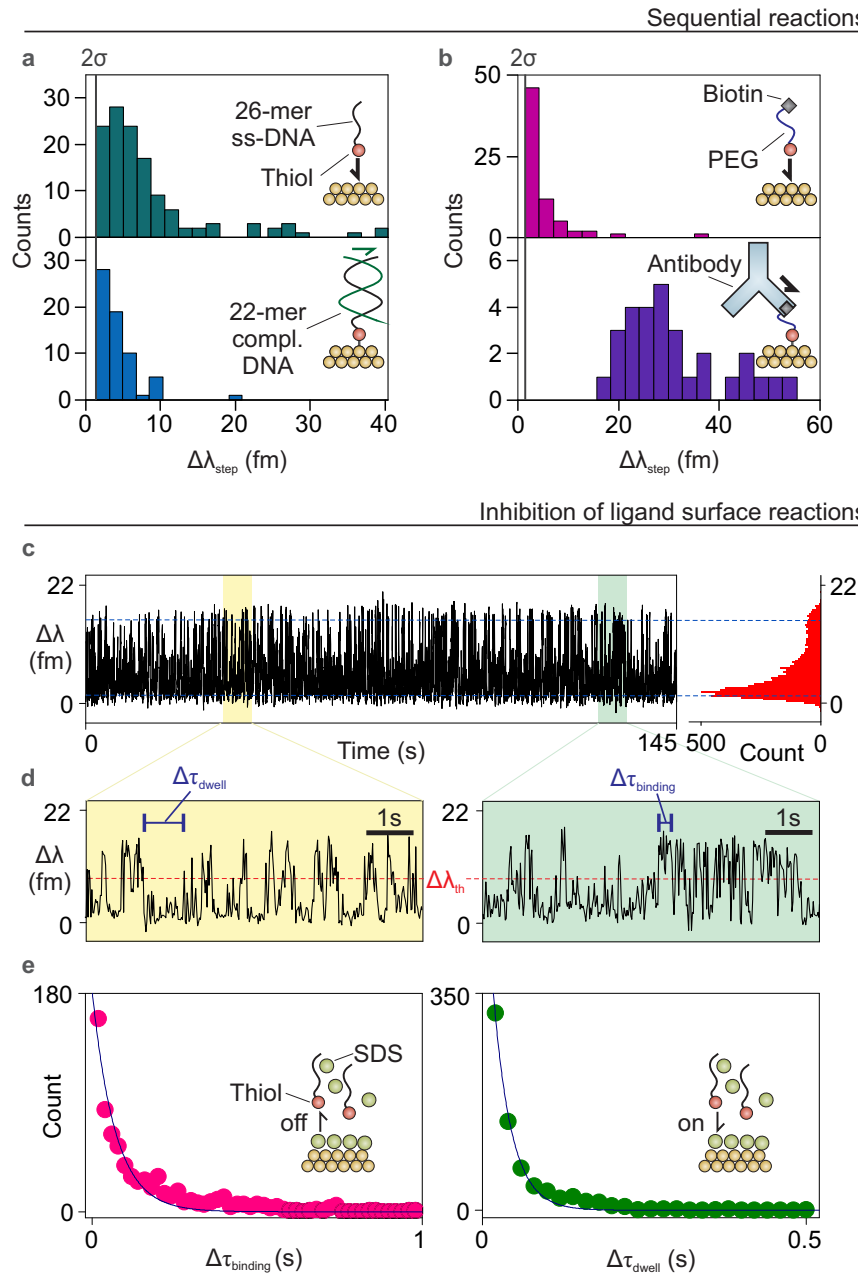

Figure 6: Specific sequential reactions and reaction kinetics in the presence of an inhibitor. Direct comparisons with step size distributions collected during the thiol-NR reaction and the ensuing, specific analyte reactions, namely (a) DNA hybridization and (b) antigen antibody binding. (c) Sample resonance trace showing a typical signal pattern from the thiol-gold reaction in the presence of SDS. (d) Detailed views of the resonance trace of (c), depicting the fluctuation of resonance position between values above and below the threshold $\lambda_{t h}$. (e) Measured distributions (points) of the durations for which the resonance position remained above $\left(\tau_{\text {binding }}\right)$ and below $\left(\tau_{\text {dwell }}\right)$ the threshold and their fitted exponential decay functions (lines). 
been pursued. Our approach serves to monitor such sequential reactions given that the detection capability is not only limited to reactions, yet is occurring directly at the NR surfaces. To demonstrate this potential, we have monitored two types of well-known biological reactions: DNA hybridization and antigen-antibody binding, after the previous modification of the NRs with thiol-linked 26-mer ss-DNA and biotinylated PEG, respectively. The affinity of the DNA hybridization depends largely on external parmeters, including the pH, electrolyte concentration, and temperature of the surrounding medium. As such we note that the mixture of transient and permanent reactions, respectively translating to spike and step signals, are under non-optimal environmental conditions, while the sole presence of permanent binding reactions occurs for high affinities between biotin and anti-biotin (Fig. 2d). Distributions of step heights observed during the 1st stage (i.e. ligand surface reaction) and the 2 nd stage (i.e. specific biological reaction) make up Fig. 6a and 6b. We find respective, average step heights of $9 \pm 4 \mathrm{fm}$ and $4 \pm 2 \mathrm{fm}$ for the DNA-thiol-gold binding and the DNA hybrization, wherein the difference is due to comparatively lower molecular mass and the larger spatial separation between the hybridizing strand and the NR surfaces (Fig. 6a). The antibody's molecular mass, 190 times higher than that of biotinylated PEG, leads to contrasting step height distributions in the case of antigen-antibody reactions (Fig. 6b). The functionality of the bound ligands can be quantitatively analyzed by comparing the step count obtained during the 1 st and the 2 nd stage of reactions. In these specific experiments, we obtained ratios of $47 \%$ and $42 \%$ for DNA hybridization over DNA-thiol gold binding and biotin anti-biodin binding over biotinylated PEG-thiol gold binding, respectively.

Another avenue for the presented method is the inspection of ligand surface reaction kinetics when a second reactant hinders or fully inhibits permanent ligand surface binding. This type of study is also not possible with ensemble based techniques as the amount of product of the reaction is extremely low in light of the existence of the inhibitor - a complication that is resolved with our approach as there is still recorded data from transient ligand surface reactions. To showcase this prospect, the thiol-NR surface interactions are 
also characterized when SDS is present. Note that SDS is a surfactant commonly used for controlling the growth directionality during nanoparticle synthesis, as its head group adsorbs to the nanoparticle surface. ${ }^{20}$ By introducing SDS, wavelength shift patterns unlike those previously discussed will appear. While we still observe a low number of step events after the injection of thiol-modified DNA oligonucleotides, the dominant type of event constitutes long intervals of spikes of equal magnitude occurring in rapid succession, interrupted by intervals devoid of events (Fig. 2e and 6c). This indicates that only few gold atoms on the surface remain accessible to the thiol groups, which may also locally compete with the SDS in order to remain immobilized on the surface and thus they undergo a rapid series of mutual exchanges with SDS until either a bond with a gold atom is formed or they depart from the sensing sites. To quantify such event patterns, we assume that, given a low ligand concentration, there are only interactions taking place at one sensing site for each event. The binding- and dwell-states are then distinguished by whether the resonance position is above or below a threshold $\Delta \lambda_{t h}$, set as half of the maximum shift (Fig. 5b). The on-rate $k_{o n}$ and the off-rate $k_{\text {off }}$ per site, extracted from the exponential decay functions fitted to the distributions of time intervals $\Delta \tau$ spent in each state (Fig. 5c), are respectively found to be 2.868 and $7.189\left(\times 10^{5} s^{-1} M^{-1}\right)$. This proves that even the efficiency of inhibitors or ligand exchange reaction kinetics can be examined using this modality.

\section{Conclusion}

A surface reaction intrinsically requires a ligand molecule to access a surface in order to form a bond. Understanding how the ligand molecule approaches the surface, starts to interact with it, and how this interaction is influenced by environmental parameters are key aspects for grasping and controlling a surface reaction in its full extent. Our method is capable of providing such information by discerning transient from permanent interactions and thus discerning ligand's access to the surface from a bond formation. We have successfully demonstrated this potential by monitoring the influence of $\mathrm{pH}$ and electrolyte concentration 
on the reaction kinetics for ligands with amine and thiol head groups in situ and with a single-molecule resolution. Over and above such functionality, critical parameters such as adsorption and desorption rates can be specified by statistical analysis of single-molecule signals. We have found that our statistically obtained values are in good agreement with the ones determined by ensemble-based methods. In addition we could directly confirm that amine groups occupy different species of surface gold atoms compared to thiol groups. The observation of reactions between different bio-functional groups tethered to the ligands and their specific counterparts does not only confirm the origin of our sensor signals, but also establishes our sensor as a tool for testing the functionality of surface modifications. We believe that our system provides a powerful tool for studying surface reactions in general as it can be extended to various types reactions, involving for example different head groups and other metals surfaces, such as aluminum, copper, or silver. Our sensing device could also probe catalytic process on metal interfaces, especially after employing faster read-out methods that are currently available. ${ }^{21,22}$

\section{Experimental details}

Technical details concerning the experiment and data analysis are outlined in the supporting information. Microsphere fabrication and nanorod deposition will, conversely, be briefly discussed here. The fused silica microspheres are formed by melting the tapered tip of a single-mode optical fiber (SMF-28, Dow Corning) with a CO2 laser $\left(\lambda_{c}=10.6 \mu \mathrm{m}\right)$. Upon heating the fiber tip close to the softening temperature of silica, it reflows as to create a sphere via surface tension. The microsphere is then immersed in an aqueous medium (Ultrapure, VWR) filtered with $0.1 \mu \mathrm{m}$ membrane filters (Merck Millipore) before the experiment and aligned until coupling to its WGMs. Microspheres with diameters of 70 to $90 \mu \mathrm{m}$ and quality factors of $10^{6}$ to $10^{7}$ are used during this step. Afterwards, gold NRs with a size of $10 \mathrm{~nm}$ x $26 \mathrm{~nm}$ and SPR at $665 \mathrm{~nm}$ are deposited at an environmental $\mathrm{pH}$ of 1.6. The binding of 
NRs is recognized as a discrete broadening of the linewidth and a simultaneous change in the resonance position. This process is monitored in real time and usually stopped when 5-7 NRs are deposited. The thiol- modified DNA oligomers used in this work have a sequence of 5'-thiol-TTTT-GAGATAAACGAGAAGGATTGAT (EuroFins MWG Operon). The typical medium condition used for thiol-modified DNA-gold binding was a $\mathrm{pH}$ of 1.8 and increasing concentrations of $\mathrm{NaCl}$ in steps of $5 \mathrm{mM}$ up to $50 \mathrm{mM}$. Interactions between thiol-modified, pegylated biotin $\left(\mathrm{C}_{34} \mathrm{H}_{65} \mathrm{~N}_{3} \mathrm{O}_{13} \mathrm{~S}_{13}\right.$, molecular mass $=788$ Da, Polypure $)$ and the gold NRs were typically performed with solutions containing $10 \mathrm{mM}$ tris- $\mathrm{HCl}$ at $\mathrm{pH} 2$ and the $\mathrm{NaCl}$ concentration was increased in steps of $2.5 \mathrm{mM}$.

\section{Acknowledgement}

The authors acknowledge financial support for this work from the Max Planck Society. E.K. and M.D.B. thank M.R. Foreman and S. Vincent for their feedback on the manuscript.

\section{Supporting Information Available}

The experimental details of plasmonic-photonic whispering gallery mode assemblies, data analysis including determination of resonance position and linewidth of WGM spectra as well as extraction of step and spike events, poisson statistics of single molecule reactions,

control experiments, history dependence of the ligand surface reactions. This material is available free of charge via the Internet at http://pubs.acs.org/.

\section{References}

(1) Sperling, R. A.; Parak, W. Philosophical Transactions of the Royal Society of London A: Mathematical, Physical and Engineering Sciences 2010, 368, 1333-1383.

(2) Grzelczak, M.; Pérez-Juste, J.; Mulvaney, P.; Liz-Marzán, L. M. Chemical Society Reviews 2008, 37, 1783-1791. 
(3) Sapsford, K. E.; Algar, W. R.; Berti, L.; Gemmill, K. B.; Casey, B. J.; Oh, E.; Stewart, M. H.; Medintz, I. L. Chemical reviews 2013, 113, 1904-2074.

(4) Indrasekara, A.; Wadams, R. C.; Fabris, L. Particle \& Particle Systems Characterization 2014, 31, 819-838.

(5) Boles, M. A.; Ling, D.; Hyeon, T.; Talapin, D. V. Nature Materials 2016, 15, 141-153.

(6) Häkkinen, H. Nature chemistry 2012, 4, 443-455.

(7) Baaske, M. D.; Foreman, M. R.; Vollmer, F. Nature nanotechnology 2014, 9, 933-939.

(8) Zijlstra, P.; Paulo, P. M.; Orrit, M. Nature nanotechnology 2012, 7, 379-382.

(9) Beuwer, M. A.; Prins, M. W.; Zijlstra, P. Nano letters 2015, 15, 3507-3511.

(10) Dubois, L. H.; Nuzzo, R. G. Annual review of physical chemistry 1992, 43, 437-463.

(11) Pong, B.-K.; Lee, J.-Y.; Trout, B. L. Langmuir 2005, 21, 11599-11603.

(12) Chen, F.; Li, X.; Hihath, J.; Huang, Z.; Tao, N. Journal of the American Chemical Society 2006, 128, 15874-15881.

(13) Karpovich, D.; Blanchard, G. Langmuir 1994, 10, 3315-3322.

(14) Marie, R.; Jensenius, H.; Thaysen, J.; Christensen, C. B.; Boisen, A. Ultramicroscopy 2002, 91, 29-36.

(15) Freeman, R. G.; Grabar, K. C.; Allison, K. J.; Bright, R. M. Science 1995, 267, 1629.

(16) Venkataraman, L.; Klare, J. E.; Tam, I. W.; Nuckolls, C.; Hybertsen, M. S.; Steigerwald, M. L. Nano Letters 2006, 6, 458-462.

(17) Kim, Y.; Hellmuth, T. J.; Burkle, M.; Pauly, F.; Scheer, E. ACS nano 2011, 5, 41044111. 
(18) Leff, D. V.; Brandt, L.; Heath, J. R. Langmuir 1996, 12, 4723-4730.

(19) Zhang, Z.; Lin, M. RSC Advances 2014, 4, 17760-17767.

(20) Das, G.; Battista, E.; Manzo, G.; Causa, F.; Netti, P. A.; Di Fabrizio, E. ACS Applied Materials \& Interfaces 2015, 7, 23597-23604.

(21) Swaim, J. D.; Knittel, J.; Bowen, W. P. Applied Physics Letters 2013, 102, 183106.

(22) Rosenblum, S.; Lovsky, Y.; Arazi, L.; Vollmer, F.; Dayan, B. Nature communications 2015, 6 . 